\title{
Fracture-Based Mesh Size Requirements for Matrix Cracks in Continuum Damage Mechanics Models
}

\author{
Frank A. Leone ${ }^{1}$ and Carlos G. Dávila ${ }^{2}$ \\ NASA Langley Research Center, Hampton, VA 23681 \\ Gerald E. Mabson ${ }^{3}$ \\ Boeing Research \& Technology, Seattle, WA 98124 \\ Madhavadas Ramnath ${ }^{4}$ \\ Boeing Commercial Airplanes, Seattle, WA 98124 \\ Imran Hyder ${ }^{5}$ \\ Boeing Research \& Technology, North Charleston, SC 29418
}

\begin{abstract}
This paper evaluates the ability of progressive damage analysis (PDA) finite element (FE) models to predict transverse matrix cracks in unidirectional composites. The results of the analyses are compared to closed-form linear elastic fracture mechanics (LEFM) solutions. Matrix cracks in fiber-reinforced composite materials subjected to mode I and mode II loading are studied using continuum damage mechanics and zero-thickness cohesive zone modeling approaches. The FE models used in this study are built parametrically so as to investigate several model input variables and the limits associated with matching the upperbound LEFM solutions. Specifically, the sensitivity of the PDA FE model results to changes in strength and element size are investigated.
\end{abstract}

\section{Introduction}

Verification and validation are critical tasks during the development of progressive damage analysis (PDA) finite element (FE) models and other predictive tools. ${ }^{1}$ Verification assesses whether a predictive analysis tool produces results that are consistent with the fundamental concepts and assumptions of the tool under evaluation, whereas validation assesses whether a predictive tool can accurately represent the physics of the problem when compared to test data. During verification exercises, predictions are generally compared to closed-form analytical solutions. Problems selected for computational tool verification are often relatively simple and isolate individual features of the tool. In the case of PDA FE models, verification problems should be selected to isolate individual deformation or failure modes.

A test case of a unidirectional, fiber-reinforced plate with a center matrix crack subjected to tensile loads (i.e., pure mode I conditions) was proposed by Mabson et al. ${ }^{2}$ to evaluate PDA FE predictions of matrix cracking. In their paper, Mabson et al. modeled this problem using the Abaqus Hashin continuum damage mechanics (CDM) model for fiberreinforced composites. ${ }^{3}$ Different combinations of matrix strength and element size were evaluated, and the results were compared to a closed-form solution based on linear elastic fracture mechanics (LEFM). Mabson et al. found that the Abaqus CDM could predict the LEFM solution only when the finite element mesh density met specific requirements based on the material properties.

In this paper, the details of a follow-on verification activity for PDA models are presented. The verification problem is a unidirectional fiber-reinforced plate with a single central matrix crack, subjected to either pure mode I or pure mode II loading. The analytical LEFM solutions for these problems are presented in Section II. Three PDA FE

\footnotetext{
${ }^{1}$ Research Aerospace Engineer, Structural Mechanics and Concepts Branch, MS 190, AIAA Member.

${ }^{2}$ Senior Research Aerospace Engineer, Structural Mechanics and Concepts Branch, MS 190, AIAA Member.

${ }^{3}$ Technical Fellow, Structures Technology, AIAA Associate Fellow.

${ }^{4}$ Lead Engineer, Structural Methods and Allowables.

${ }^{5}$ Engineer, Structural Analysis and Tools.
} 
models are evaluated: the Abaqus CDM model for fiber-reinforced composites, ${ }^{3}$ CompDam, ${ }^{4,5}$ and Abaqus zerothickness cohesive elements. Details of the PDA models and their implementation in FE models of the verification problem are presented in Section III. Parametric studies are presented in Section IV in which the PDA FE model inputs of matrix strength and element size are varied to investigate under what conditions LEFM and PDA FE solutions agree, and to investigate the mesh size requirements of each case and how they relate to material inputs. A comparison of the LEFM and PDA FE results is presented in Section II, with further discussion of the results in Section VI.

\section{Linear Elastic Fracture Mechanics Solutions}

The analytical LEFM solution for the fracture of an infinite plate with a central matrix crack under plane stress conditions can be written as a function of the orthotropic stiffness properties, the initial crack half-length $a_{0}$, and the material fracture toughness. The far-field normal stress $\sigma_{\infty, c}$ at which the central matrix crack is predicted to propagate under mode I deformation may be expressed as:

$$
\sigma_{\infty, c}=\sqrt{\frac{G_{I c}}{\pi a_{0}}}\left[\frac{4 G_{x y} E_{x} E_{y}^{3 / 2}}{2 G_{x y} E_{x}^{1 / 2}+E_{x} E_{y}^{1 / 2}-2 v_{x y} G_{x y} E_{y}^{1 / 2}}\right]^{1 / 4}
$$

where $x$ indicates the direction parallel to the crack, $G_{I c}$ is the mode I fracture toughness, and $E_{x}, E_{y}, G_{x y}$, and $v_{x y}$ are the orthotropic stiffness properties. ${ }^{2}$ Similarly, the far-field shear stress $\tau_{\infty, c}$ at which the central matrix crack is predicted to propagate under mode II deformation has the form:

$$
\tau_{\infty, c}= \pm \sqrt{\frac{G_{I I c}}{\pi a_{0}}}\left[\frac{4 G_{x y} E_{x}^{2} E_{y}^{1 / 2}}{2 G_{x y} E_{x}^{1 / 2}+E_{x} E_{y}^{1 / 2}-2 v_{x y} G_{x y} E_{y}^{1 / 2}}\right]^{1 / 4}
$$

where $G_{I I c}$ is the mode II fracture toughness values.

It can be observed that the failure stresses given by Equations (1) and (2) are independent of the material strengths ( $\sigma_{c}$ for mode I and $\tau_{c}$ for mode II) and the element size $L_{e}$. It is of interest to study how these additional independent model variables affect the agreement between LEFM and PDA FE models. The LEFM solutions in Equations (1) and (2) serve as upper bounds for valid PDA FE predictions of far-field failure stress.

\section{Progressive Damage Analyses}

To re-create the studies of Mabson et al., ${ }^{2}$ it is necessary to perform many FE analyses in which a single variable is swept through a range of values. As a result, the PDA FE models in this study were built using the Abaqus Scripting Interface to more easily vary the model inputs (i.e., plate width $W$, plate height $H$, initial crack length, element size $L_{e}$, material properties, etc.), and to more easily submit and post-process large batch jobs. Plane stress conditions were assumed, and reduced-integration, plane stress CPS4R elements with hourglass control were used to model the centercracked plates in each model.

Three PDA FE models are used in this study: the built-in Abaqus CDM model for fiber-reinforced composites, ${ }^{3}$ CompDam, ${ }^{4,5}$ and Abaqus COH2D4 cohesive elements. The first model, the Abaqus CDM model, is a typical strainsoftening CDM method, in which the presence of damage is represented by softening terms of the constitutive stiffness matrix that are associated with certain damage modes (i.e., decreasing the matrix-direction stiffness after matrix damage initiation). The second model, CompDam, is a composite CDM material model developed by researchers in the Structural Mechanics and Concepts Branch at NASA Langley Research Center. The CompDam material model utilizes the deformation gradient decomposition (DGD) method $^{5}$ to represent matrix cracks. The DGD method involves embedding a cohesive crack in a bulk material continuum, and separately tracking the orientation and opening of the cohesive crack and the deformation of the bulk material continuum. The third model, Abaqus zero-thickness cohesive elements, utilizes a bilinear traction-separation response to represent the damage initiation and evolution.

A schematic of the model configuration for the two CDM models is shown in Figure 1. The majority of the model area is defined using a linear elastic orthotropic material model. The CDM models are applied only in the refined mesh region that surrounds the crack. For the cohesive element models, a single row of zero-thickness cohesive elements is inserted along the crack line, and a linear elastic orthotropic material model is used throughout the rest of the model.

For the Abaqus CDM and cohesive element models, the initial crack is represented by artificially reducing the strength and fracture toughness material properties for the elements within the crack. The reduced strength is equal to the nominal strength multiplied by a factor of $10^{-3}$, and the reduced fracture toughness is equal to the nominal fracture 
toughness multiplied by a factor of $10^{-6}$. For CompDam, the initial crack is represented by setting the matrix damage state variable to 1.0 (i.e., fully failed) as an initial condition.

For mode I loading, the nodes at the top-left and bottom-left corners of the model are fixed in the 1-direction. The nodes along the top edge are displaced in the positive 2-direction and the nodes along the bottom edge are displaced in the negative 2-direction. For mode II loading, the 1- and 2- direction displacements along all outer edges of the model are defined according to pure shear deformation through an analytical field.

Because the current implementation of CompDam is compatible only with Abaqus/Explicit, all analyses in this study were performed using Abaqus/Explicit 6.14-1. A uniform mass scaling factor of $10^{4}$ was applied. Displacements were applied with an S-curve amplitude over a period of 0.2 second in a single geometrically nonlinear solution step. The step parameters were chosen to approximate quasi-static loading conditions. The model-wide strain energy and kinetic energy were tracked and compared to confirm that quasi-static conditions were achieved.

For each PDA FE analysis, the far-field stresses at which damage initiates ( $\sigma_{\infty, i}$ for mode I and $\tau_{\infty, i}$ for mode II) and final failure occurs ( $\sigma_{\infty, f}$ for mode I and $\tau_{\infty, f}$ for mode II) were automatically extracted. Here, damage initiation is defined as when the integration points of an element immediately ahead of the initial crack tips have a nonzero matrix damage state variable. Final failure is defined as the onset of unstable damage propagation. In a well-designed PDA FE model of the center-cracked infinite plate, unstable damage propagation would occur when the matrix damage state variable in an element immediately ahead of the original crack tip reaches the value of 1.0. In order to account for unexpected directions and rates of damage propagation when evaluating certain combinations of model inputs, a group of elements some distance ahead of the initial crack tips was selected to define when unstable failure propagation occurs.

\section{Parametric Study}

Parametric studies were conducted for each of the three damage models (Abaqus CDM, CompDam, and cohesive elements) for both mode I and mode II loading conditions. In each parametric study, either the matrix strength or the element size in the vicinity of the initial crack is varied. The output of each parametric study is a comparison of the LEFM solution (from Section II) with the far-field stresses at which unstable matrix crack growth occurs as either the strength or element size varies in the PDA FE analyses. With this approach, upper bounds can be established for the matrix strength and element size required for the PDA models to match LEFM predictions.

For all of the analyses, the plate width $W$ is kept constant at $127 \mathrm{~mm}$, and the crack half-length $a_{0}$ is kept constant at $12.7 \mathrm{~mm}$. The height of the refined region around the crack is $3.0 \mathrm{~mm}$. Material properties from literature for IM7/8552 are used, and are summarized in Table $1 .{ }^{6}$ All elements in the vicinity of the crack have an aspect ratio of 1.0, i.e., equal element size in the 1-direction and 2-direction.

For the analyses in which the matrix strength is varied, the element size is held constant at $0.2 \mathrm{~mm}$. Results are plotted against a strength factor, defined as the matrix strength used in the analysis divided by the nominal matrix strengths $\sigma_{c}$ and $\tau_{c}$ in Table 1 . For the analyses in which the element size is varied, only the element size in the vicinity of the crack is changed, and the nominal matrix strengths in Table 1 are used.

Table 1. IM7/8552 material properties. ${ }^{6}$

\begin{tabular}{clrl}
\hline Symbol & Description & Value & Units \\
\hline$E_{x}$ & Young's modulus, fiber & 171,420 & $\mathrm{MPa}$ \\
$E_{y}$ & Young's modulus, matrix & 9080 & $\mathrm{MPa}$ \\
$G_{x y}$ & Shear modulus & 5290 & $\mathrm{MPa}$ \\
$v_{x y}$ & Poisson ratio & 0.32 & - \\
$\sigma_{c}$ & Strength, mode I & 62.3 & $\mathrm{MPa}$ \\
$\tau_{c}$ & Strength, mode II & 92.3 & $\mathrm{MPa}$ \\
$G_{I c}$ & Fracture toughness, mode I & 0.277 & $\mathrm{~kJ} / \mathrm{m}^{2}$ \\
$G_{I I c}$ & Fracture toughness, mode II & 0.788 & $\mathrm{~kJ} / \mathrm{m}^{2}$ \\
\hline
\end{tabular}

The computational costs of the analyses varied with the different combinations of model inputs. However, as a point of reference, the analysis using the Abaqus CDM model, an element size of $0.2 \mathrm{~mm}$, nominal matrix strength properties, and mode I loading conditions had 224,720 degrees of freedom, and took approximately 28 minutes to complete using 12 processors. 


\section{Results}

\section{A. Mode I}

For an infinite plate subjected to mode I loading with an initial crack length of $25.4 \mathrm{~mm}$, the analytical LEFM solution predicts failure at a far-field normal stress of $9.3 \mathrm{MPa}$. The results of the analyses in which the matrix strength is varied for the Abaqus CDM, CompDam, and cohesive element models are shown in Figures 2, 3, and 4, respectively. The analyses with each of the three damage models yield similar results, and these results are similar to those published in reference 2 . As expected, the far-field stress at initiation $\sigma_{\infty, i}$ increases linearly with the strength factor, and the farfield stress at final failure $\sigma_{\infty, f}$ forms a plateau that corresponds to the LEFM solution. The far-field stress plateaus slightly overestimate the LEFM solution due to the finite size of the modeled plate. Increasing the height $H$ of the model and using the displacement boundary conditions for load application shifts the final failure curves in Figures 2 and 3 downward toward the LEFM solution. In order to maintain reasonable computational run times, $H$ was kept equal to $2 W$ for the mode I problems. When $\sigma_{\infty, i}$ is equal to the LEFM prediction (i.e., strength factor equal to 1.6 in Figures 2 and 3, and 1.2 in Figure 4), the failure becomes brittle, i.e., failure initiation and final failure of the panel coincide. As the strength factor continues to increase, the PDA FE analyses increasingly overpredict the LEFM solution. These results that overpredict LEFM are incorrect and do not accurately represent the physics of the problem.

In addition to the far-field stresses corresponding to damage initiation and final failure, the predicted and observed fracture process zone lengths are also plotted in Figures 2, 3, and 4. The length of the fracture process zone for a mode I crack in an orthotropic material under plane stress conditions has been derived by Turon et al. ${ }^{7}$ as:

$$
l_{\mathrm{FPZ}}=\gamma \frac{E_{y}}{Q} \frac{G_{I c}}{\sigma_{c}^{2}}
$$

where $Q$ is a function of the orthotropic stiffness:

$$
Q=\frac{1}{2} \sqrt{2\left(\sqrt{\frac{E_{y}}{E_{x}}}-v_{y x}\right)+\frac{E_{y}}{G_{x y}}}
$$

and the nondimensional parameter $\gamma$ is assumed to be equal to 0.884 , based on the work of Rice ${ }^{8}$ and the observations of Turon et al. ${ }^{9}$ Using the material properties in Table 1, Equations (3) and (4) yield $l_{\mathrm{FPZ}} \approx 0.8 \mathrm{~mm}$ for mode I loading. The length of the fracture process zone observed in the models is here defined as the length of the group of elements ahead of the crack tip with a damage variable greater than zero and fewer than one immediately before final failure. The lengths of the fracture process zones observed in all three models agree well with Equation (3). The trend of $\sigma_{\infty, f}$ smoothly approaching the LEFM prediction is disrupted when $l_{\mathrm{FPZ}}<2 L_{e}$ for the CDM models, and when $l_{\mathrm{FPZ}}<3 L_{e}$ for the cohesive element models. The results with these coarsely represented fracture process zones are highlighted in each of the results figures. The strength-dependent failure predictions for each of the models occur when $l_{\mathrm{FPZ}}<1$, i.e., the damage state variable in the element ahead of the existing damage changes directly from zero to one. These observations are in agreement with Turon's recommendation to have at least three cohesive elements within the fracture process zone for a mode I crack. ${ }^{9}$

The results of the analyses in which the strength is kept constant and the element size in the vicinity of the crack is varied for the Abaqus CDM, CompDam, and cohesive element models are shown in Figures 5, 6, and 7, respectively, for mode I loading. The effect of increasing the element size is similar to the effect of increasing the strength: increasing the size of the elements delays the initiation of damage up to an apparent critical element size, above which only erroneous, mesh-dependent failures occur. For analyses with elements smaller than this critical size, the prediction of the final failure of the plate correlates well with the LEFM solution. This critical element size can be defined as the element size when $\sigma_{\infty, i}$ is equal to $\sigma_{\infty, c}$ in Figures 5, 6, and 7. The predicted and observed $l_{\mathrm{FPZ}}$ are not included in Figures 5, 6, and 7 as the predicted $l_{\mathrm{FPZ}}$ is constant and the observed $l_{\mathrm{FPZ}}$ values are constant, though more coarsely approximated as the element size increases and there are fewer elements within the process zone. Regions in which the process zone is too coarsely represented and in which mesh-dependent failures occur are highlighted in each of the results figures. 


\section{B. Mode II}

An infinite plate subjected to pure shear deformation with an initial crack length of $25.4 \mathrm{~mm}$ and the material properties listed in Table 1 is predicted to fail at a far-field shear stress of $\pm 32.6 \mathrm{MPa}$ according to Equation (2). The results for the PDA FE analyses in which the matrix strength is varied are shown in Figures 8, 9, and 10.

Each of the models exhibits a ductile failure mode for lower strength factors, in which the length of the process zone is large with respect to the initial crack length. Eventually, the full width of the plate is within the developing process zone (i.e., $\left.W<2\left(a_{0}+l_{\mathrm{FPZ}}\right)\right)$. As the crack begins to interact with the panel edges, the assumption of an infinite plate is invalidated, and the LEFM predictions in Equations (1) and (2) are unable to match the PDA FE results.

Similar to the mode I results, $\tau_{\infty, i}$ increases linearly with strength factor in the CompDam and cohesive element models. The $\tau_{\infty, i}$ results for the Abaqus CDM model, however, exhibit a positive parabolic trend with increasing strength factor. While the CompDam and cohesive element models exhibit ductile failure only for strength factors of 0.2 and less, the Abaqus CDM models exhibit ductile failure up to a strength factor of 0.7. The Abaqus CDM $\tau_{\infty, f}$ results also significantly overpredict the LEFM solution for intermediate strength factors, while the CompDam and cohesive element $\tau_{\infty, f}$ results gradually approach the LEFM prediction, similar to the mode I results. The Abaqus CDM and CompDam analyses both become strength-dependent at a strength factor of approximately 1.5 (138 MPa). The cohesive element failures are strength-dependent for strength factors greater than 4.0.

The length of the fracture process zone for a mode II crack in an orthotropic material under plane stress conditions has been derived by Turon et al. ${ }^{7}$ as:

$$
l_{\mathrm{FPZ}}=\gamma \frac{E_{y}}{Q} \sqrt{\frac{E_{x}}{E_{y}}} \frac{G_{I I c}}{\tau_{c}^{2}}
$$

Using the same assumption that $\gamma$ is equal to 0.884 and the material properties in Table $1, l_{\mathrm{FPZ}} \approx 4.4 \mathrm{~mm}$ for mode II loading. The observed fracture process zone lengths in the cohesive element models in Figure 10 slightly underestimate the predictions of Equation (5), but follow the same trend. The observed fracture process zone lengths for the Abaqus CDM (Figure 8) and CompDam (Figure 9) models significantly underestimate the predictions of Equation (5). As the number of elements within the process zone decreases in the CDM analyses, the fracture process zone becomes more coarsely represented, and the element size begins to affect damage propagation. For the CDM analyses, when there are less than six elements within the process zone, the first element to fully fail is not at the tail of the fracture process zone, but is instead located one element within the process zone, leaving a one-element bridge at the initial crack tip, as shown in Figure 11a. This "inner process zone element failure" behavior occurs in the highlighted "coarse process zone" region in Figures 8 and 9. As the number of elements within the process zone decreases further, non-self-similar damage propagation is observed, wherein elements not aligned with the initial crack fail and crack branching sometimes occurs, as shown in Figure 11b. Non-self-similar crack growth blunts the crack and increases its apparent toughness. Like the mode I results, each of the models exhibits brittle, strength-dependent failure when the observed $l_{\mathrm{FPZ}}$ is less than $L_{e}$.

The results obtained by holding the matrix strength constant and varying the element size are shown in Figures 12, 13, and 14 for the Abaqus CDM, CompDam, and cohesive element models, respectively. The Abaqus CDM results fail to match the LEFM predictions for any element size. For the CompDam and cohesive element models, increasing the size of the elements delays the initiation of damage up to an apparent critical element size. Like the mode I results, this critical element size can be defined as the intersection of the damage initiation and LEFM curves in Figures 13 and 14. Below this critical element size, the far-field stress at final failure is relatively constant. For elements larger than this critical size, the prediction of far-field stress at final failure is a function of element size, i.e., mesh-dependent. While the predicted $l_{\mathrm{FPZ}}$ is constant for different element sizes, the mode II CDM results exhibit decreasing observed $l_{\mathrm{FPZ}}$ with increasing $L_{e}$. For $L_{e}$ greater than $0.25 \mathrm{~mm}$, the CompDam results begin to exhibit inner process zone element failure and non-self-similar crack growth behavior illustrated in Figure 11.

\section{Discussion}

The maximum size of finite elements that can be used with CDM material models has been erroneously assumed to be determined by snap-back criteria. ${ }^{10}$ These snap-back criteria ensure that when damage initiates, the elastic strain energy in an individual element has not exceeded the fracture energy. To avoid snap-back associated with matrix cracking, the condition: 


$$
L_{e} \leq \frac{2 G_{I c} E_{y}}{\sigma_{c}^{2}}
$$

must be satisfied for mode I, and the condition:

$$
L_{e} \leq \frac{2 G_{I I c} G_{x y}}{\tau_{c}^{2}}
$$

must be satisfied for mode II. For the material properties in Table 1, these snap-back limits are, respectively, $1.30 \mathrm{~mm}$ and $0.98 \mathrm{~mm}$ for the mode I and mode II matrix failure modes. While these snap-back limits ensure that elements are sized so that each element is individually capable of dissipating the correct amount of energy, they do not ensure that the elements are capable of working together to model a larger fracture process. The results presented in this paper confirm the finding in reference 2 that the element size requirements that must be met for CDM analyses are more restrictive than the snap-back limits. Using element sizes larger than these additional limits results in predictions that are mesh-dependent, and yields predictions that are erred in terms of failure stress, damage size, and damage propagation direction.

For mode I loading, the Abaqus CDM and CompDam analyses indicate that ensuring that the element size is smaller than the predicted $l_{\mathrm{FPZ}}$ avoids mesh-dependent far-field failure stress predictions. However, having at least two elements within the fracture process zone yields results that better correlate with the far-field failure stress predicted by LEFM and that are better converged in terms of damage size, shape, path, etc. The cohesive element analyses yield similar findings: that having less than two elements within the predicted $l_{\text {FPZ }}$ avoids mesh-dependent predictions, but analysis results are converged in terms of failure load and damage propagation when there are at least three elements within the fracture process zone.

For mode II loading, the Abaqus cohesive element analyses avoid mesh-dependent inaccuracies as long as the element size is less than the predicted $l_{\mathrm{FPZ}}$. However, the results better correlate with LEFM in terms of far-field failure stress and smoother damage propagation is observed only when at least two elements are within the fracture process zone. For the mode II CDM analyses, the model fracture process zone lengths do not correlate well with the analytical solutions, and a limit on element size cannot be defined in terms of the analytical $l_{\mathrm{FPZ}}$. In terms of the $l_{\mathrm{FPZ}}$ in the mode II CompDam analyses, at least four elements must be in the process zone to avoid brittle, mesh-dependent failure, and more than six elements are required for smooth damage propagation and good correlation with LEFM.

There is a large difference in the mode II element size requirements for the CDM and cohesive element models. The reason for this difference is related to the element size across the crack, i.e., along the 2-direction in Figure 1. In the cohesive element analyses, the width of the initial crack is zero, while the width of the crack in the CDM analyses is equal to the element size. As the element size increases, the crack effectively becomes blunter due to CDM models smearing the crack and material stress states. A blunt crack has a lower stress concentration factor associated with it, and initiates damage later than a sharper crack under the same conditions. Increasing the element size across the crack delays damage initiation, eventually leading to brittle, mesh-dependent predictions. If one were to decrease the element size across the crack to a limit of zero, the CompDam results would approach the cohesive element results, as both CompDam and cohesive elements represent damage with a bilinear traction-separation law.

The location of the peak shear stress ahead of a notch loaded in mode II is a function of the notch width. With an element aspect ratio of 1.0, the CDM models consistently predict shear damage initiation in the second element ahead of the crack, rather than the element immediately ahead of the crack, as occurs in the mode II cohesive element models. For increasingly brittle failure processes, this leads to the observed instances of inner process zone element failure ahead of the initial crack in the mode II CDM models. In order to avoid this mode of damage propagation in the mode II CompDam analyses, more than six elements are required in the observed fracture process zone.

The pre-crack in all of the analyses presented in this paper is represented using the damage model under evaluation. That is, the elements within the pre-crack are not removed, but are elements containing damage. It is important to study a damage model's ability to propagate a crack from its own representation of damage rather than a meshed discontinuity, as once damage propagates any amount, the damage model must be able to continue to propagate the damage accurately.

The overprediction of the far-field stress corresponding to final failure by the Abaqus CDM model for mode II loading conditions is characteristic of strain-softening CDM methods in geometrically nonlinear analyses. The large shear deformation applied to cracked material points results in a rotation of the local material directions. Calculating a strain in this rotated, smeared material system has been found to cause unintended load transfer across matrix cracks by incorrectly tracking the orientation of the fibers. The use of the deformation gradient decomposition method for crack definition and finite stress and strain definitions for the bulk material in CompDam allows for better tracking of 
matrix crack orientation under large shear deformation and, when the mesh is sufficiently refined, accurately predicts mode II matrix cracking. ${ }^{5}$

\section{Closing Remarks}

The ability for CDM and cohesive element models to predict transverse matrix cracks in a unidirectional fiberreinforced composite material under tensile and pure shear loading conditions has been evaluated. It was found that an additional set of fracture-based element size limits exists. Not accounting for these fracture-based element size limits causes erroneous PDA FE analysis results which overestimate the upper-bound LEFM predictions.

The snap-back limits and the fracture-based limits on element size observed in this paper are upper limits, not a recommended element size. The progressive damage models used in this study are all intended to soften material gradually and to dissipate the correct amount of energy, not to have their mesh sized in order to achieve sudden failure for a single load case and/or failure mode. Performing an analysis at these limits of sudden erroneous failure produces unwanted dynamic effects in quasi-static explicit analyses and convergence issues in implicit analyses. In addition, having damage state variables be only either zero or one causes stress fields ahead of modeled damage to be more dependent on the finite element discretization than when fracture process zones are properly modeled and distributed across several elements.

There is a real, practical need to size elements as large as possible to reduce the sometimes intractable computational run times of progressive damage finite element analyses. However, using inappropriately large elements when modeling damage can lead to predictions that are inaccurate in terms of failure stress, damage mode, damage size, and direction of damage propagation. Care must be taken to ensure that elements are sized to predict failure accurately both individually and as part of a fracture process zone.

\section{References}

${ }^{1}$ Thacker, B., "The Role of Nondeterminism in Verification and Validation of Computational Solid Mechanics Models," SAE Technical Paper 2003-01-1353, 2003.

${ }^{2}$ Mabson, G. E., Weckner, O., and Ramnath, M., "Finite Element Based Decohesive Failure Simulation Sensitivity Studies," 53 ${ }^{\text {rd }}$ AIAA/ASME/ASCE/AHS/ASC Structures, Structural Dynamics and Materials Conference, AIAA Paper 2012-1622, April 23-26 2012, Honolulu, HI.

${ }^{3}$ ABAQUS, Abaqus 6.14 Online Documentation, Dassault Systèmes, Providence, RI, 2014.

${ }^{4}$ Rose, C. A., Dávila, C. G., and Leone, F. A., "Analytical Methods for Progressive Damage of Composite Structures," NASA/TM-2013-218024, NASA Langley Research Center, Hampton, VA, July 2013.

${ }^{5}$ Leone, F. A., "Deformation Gradient Tensor Decomposition for Representing Matrix Cracks in Fiber-Reinforced Materials," Composites Part A: Applied Science and Manufacturing, Vol. 76, 2015, pp. 334-341.

${ }^{6}$ Camanho, P. P., Maimí, P., and Dávila, C. G., "Prediction of Size Effects in Notched Laminates Using Continuum Damage Mechanics," Composites Science and Technology, Vol. 67, 2007, pp. 2715-2727.

${ }^{7}$ Turon, A., Costa, J., Camanho, P. P., and Maimí, P., "Analytical and Numerical Investigation of the Length of the Cohesive Zone in Delaminated Composite Materials,” in Camanho, P. P., Dávila, C. G., Pinho, S. T., and Remmers, J. J. C. (eds) Mechanical Response of Composites. Springer, 2008, pp. 77-97.

${ }^{8}$ Rice, J. R., "The Mechanics of Earthquake Rupture," in Dziewonski, A. M., Boschi, E. (eds) Physics of the earth's interior, Proceedings of the International School of Physics 'Enrico Fermi'. Italian Physical Society and North-Holland Publ Co., pp. 555-649.

${ }^{9}$ Turon, A., Dávila, C. G., Camanho, P. P., Costa, J., “An Engineering Solution for Mesh Size Effects in the Simulation of Delamination using Cohesive Zone Models,” Engineering Fracture Mechanics, Vol. 74, 2007, pp. 1665-1682.

${ }^{10}$ Maimí, P., Camanho, P. P., Mayugo, J. A., Dávila, C. G., “A Continuum Damage Model for Composite Laminates: Part II Computational Implementation and Validation," Mechanics of Materials, Vol. 39, 2007, pp. 909-919. 


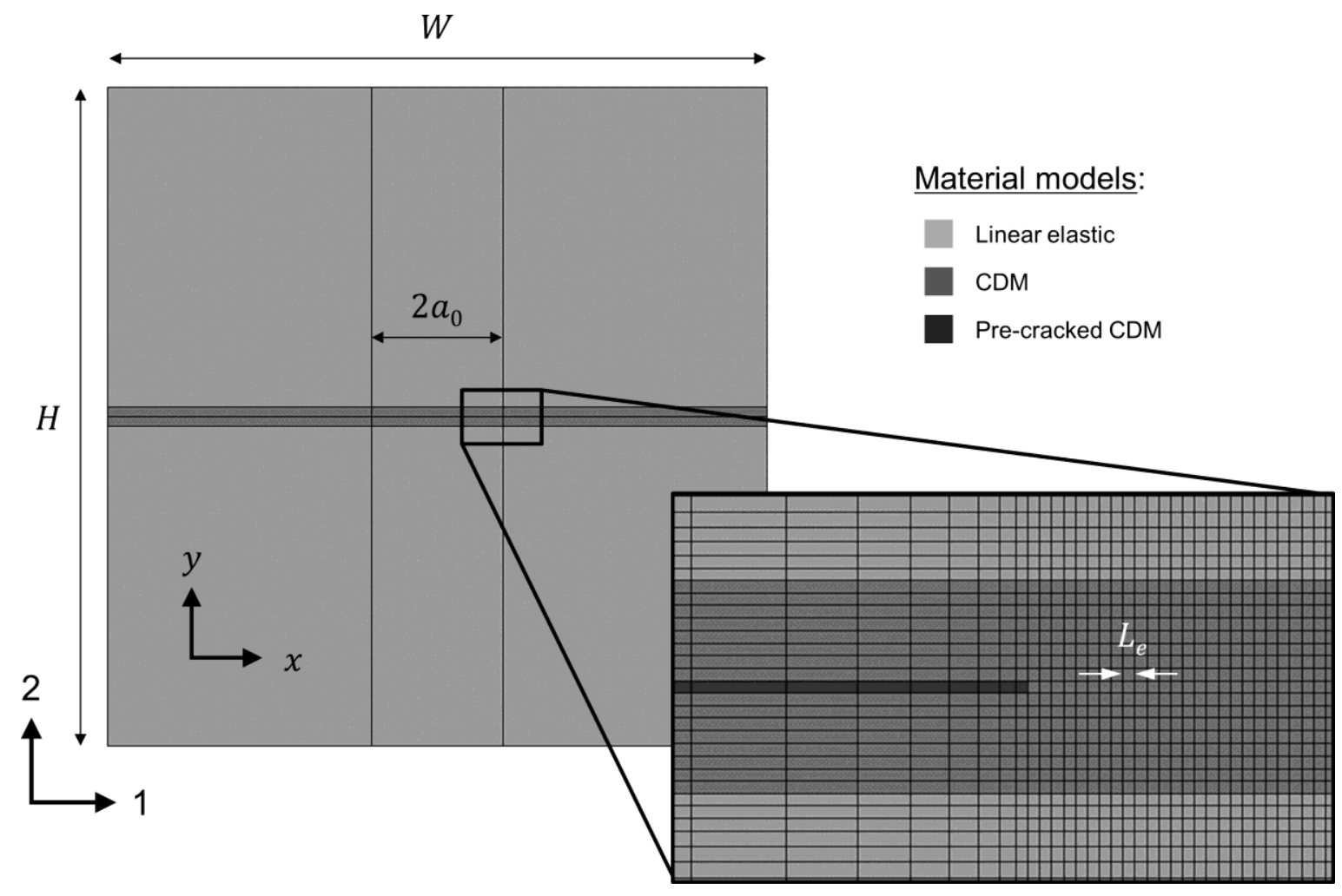

Figure 1. PDA FE model schematic.

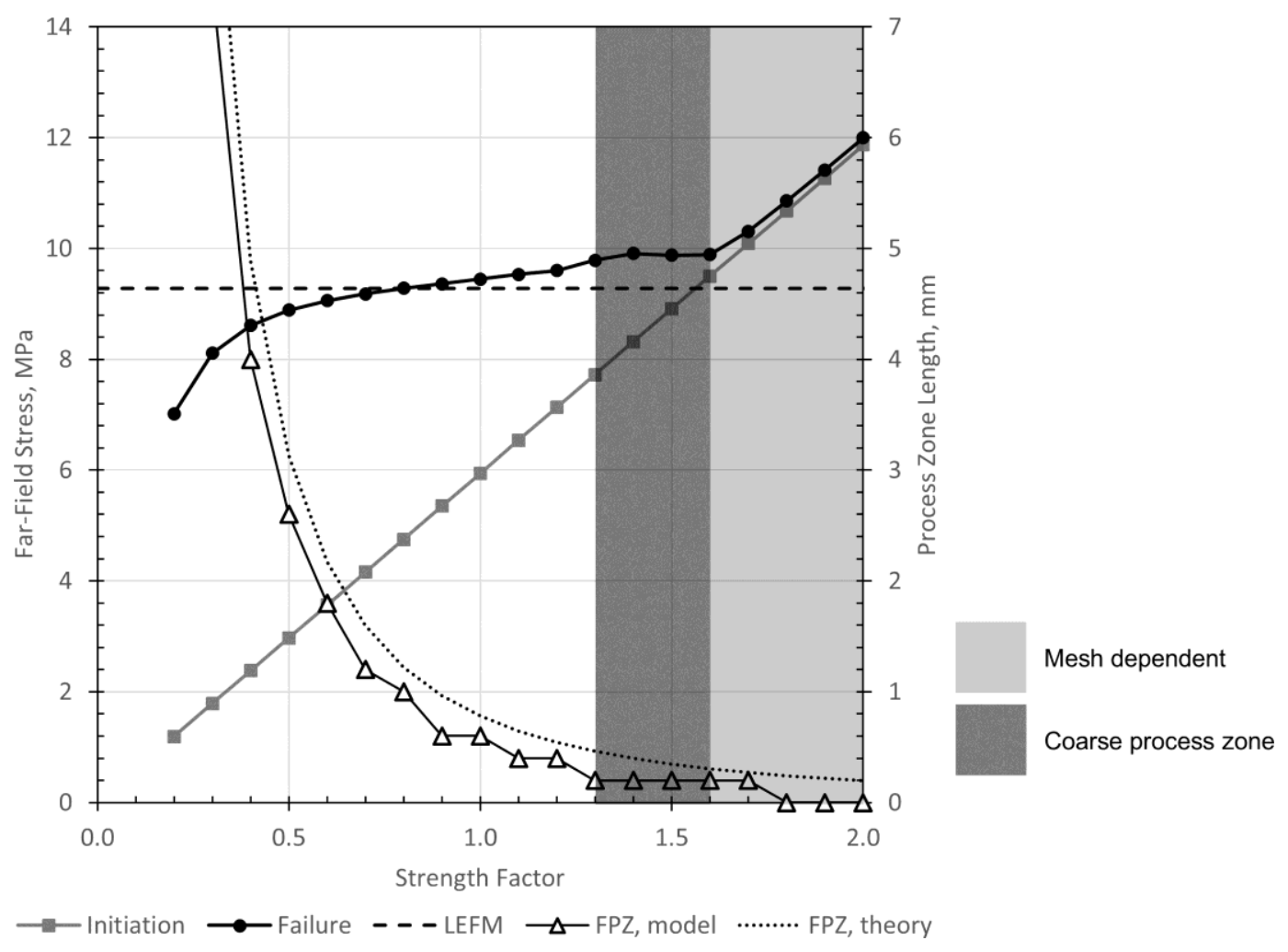

Figure 2. Abaqus CDM model predictions for mode I loading, varying the strength factor. 


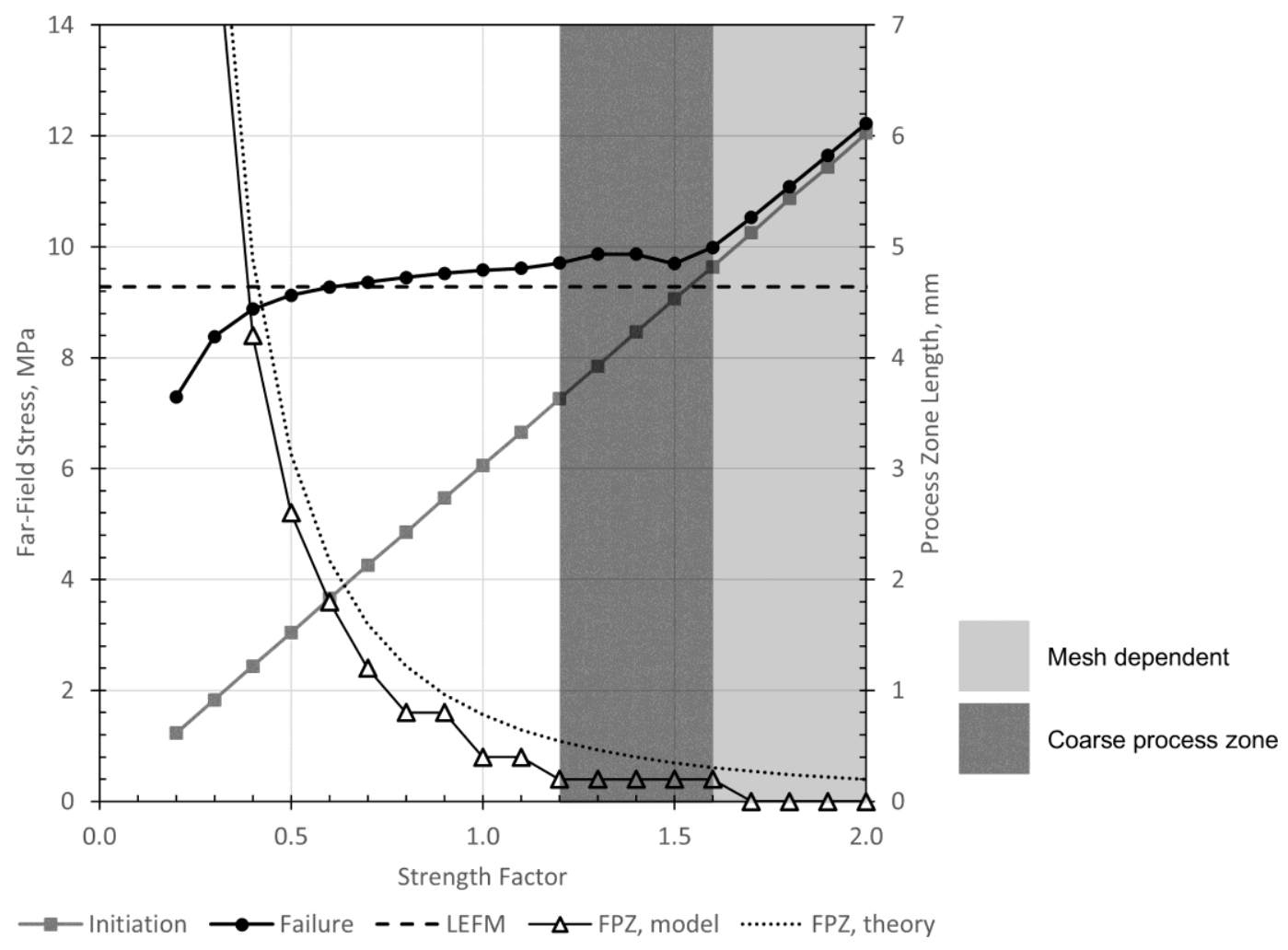

Figure 3. CompDam predictions for mode I loading, varying the strength factor.

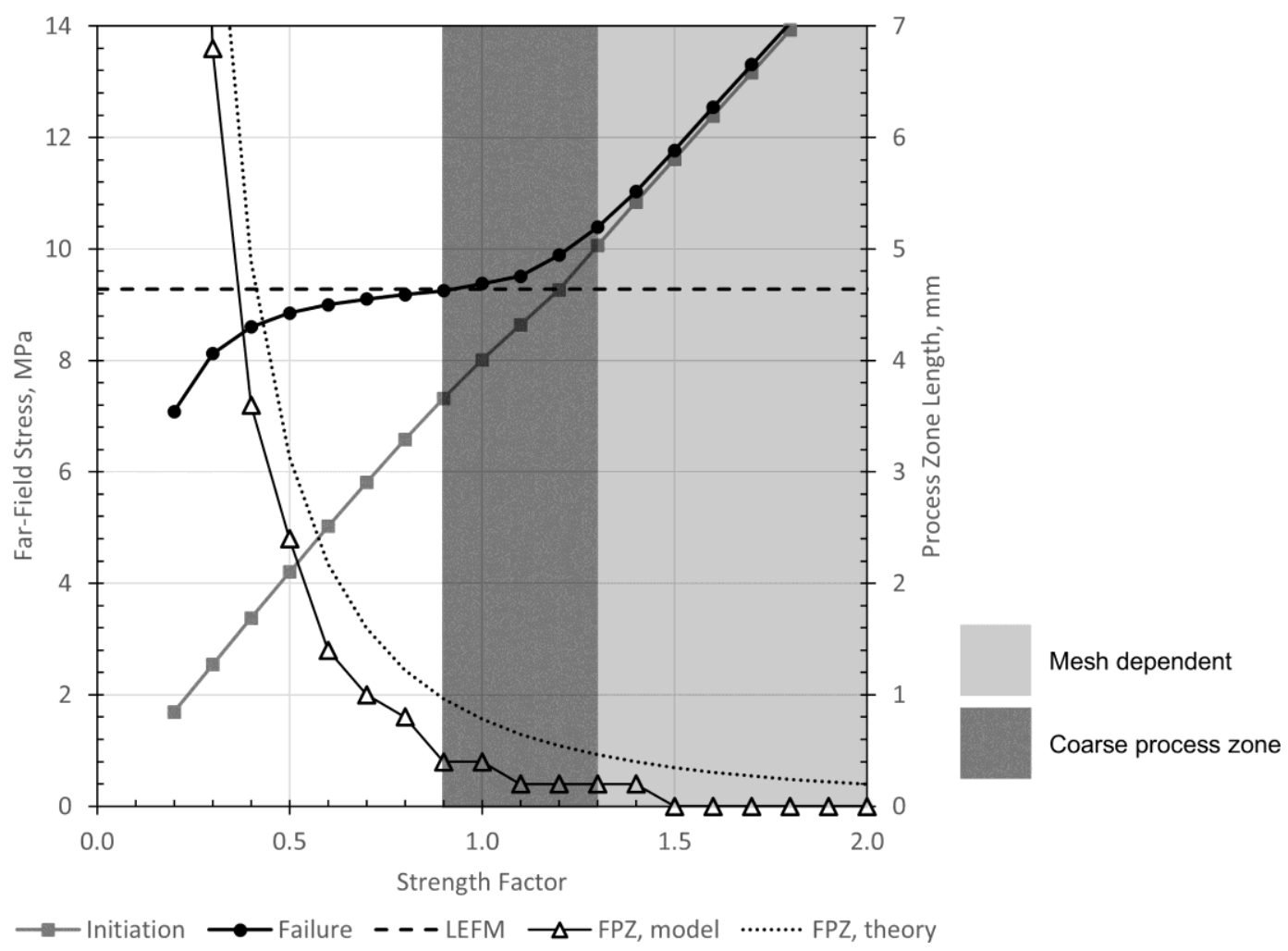

Figure 4. Cohesive element predictions for mode I loading, varying the strength factor. 


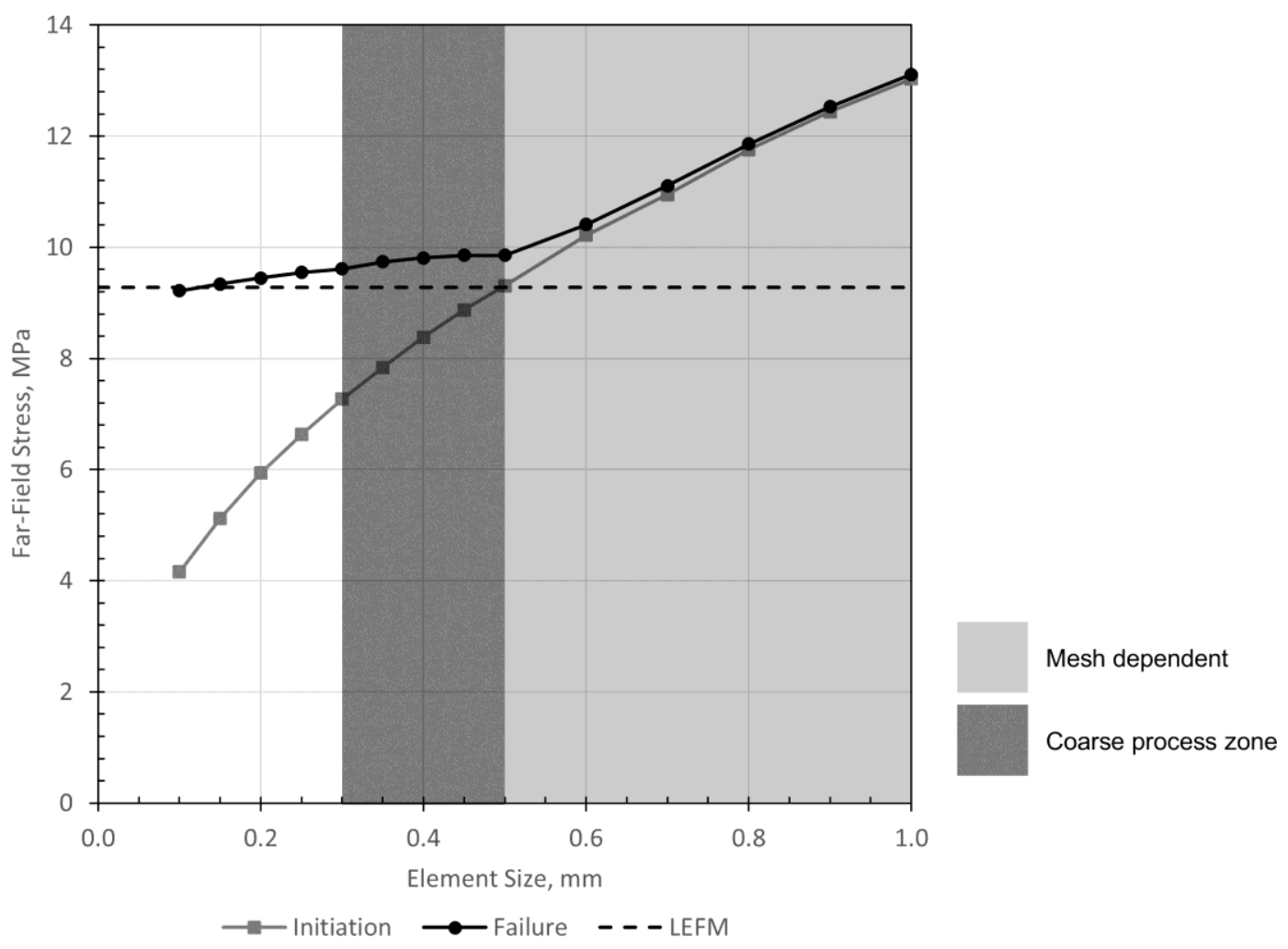

Figure 5. Abaqus CDM predictions for mode I loading, varying the element size.

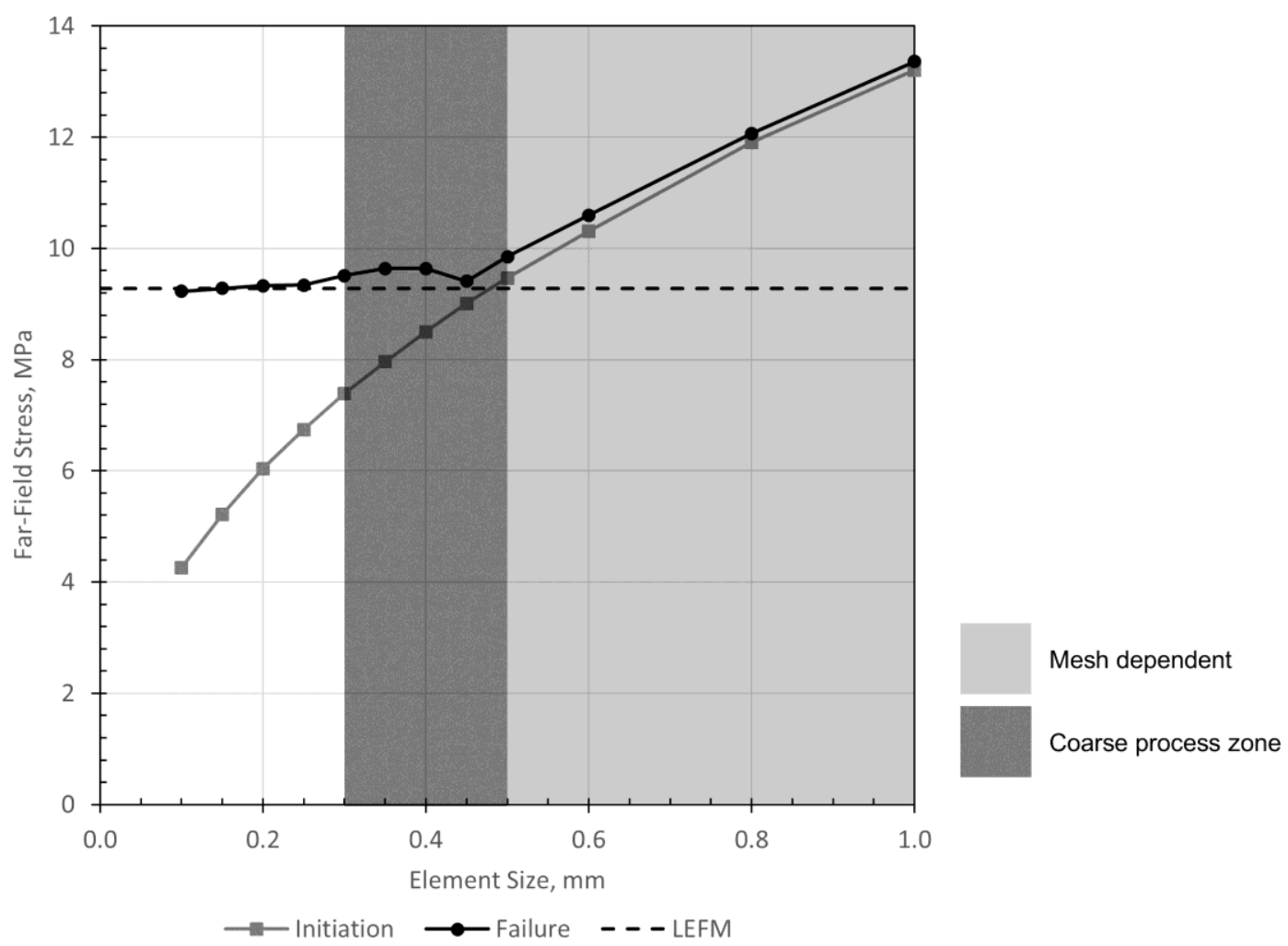

Figure 6. CompDam predictions for mode I loading, varying the element size. 


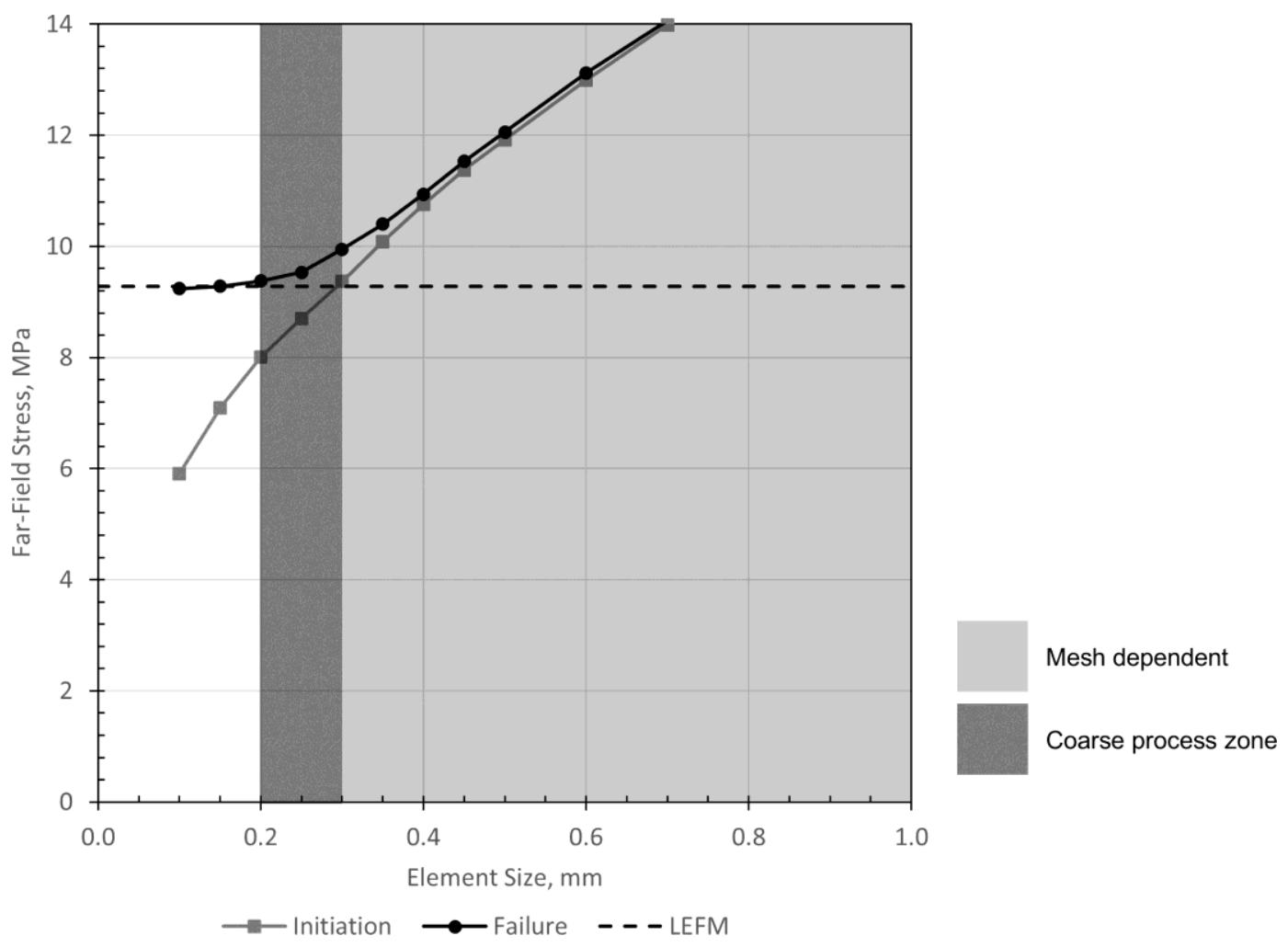

Figure 7. Cohesive element predictions for mode I loading, varying the element size.

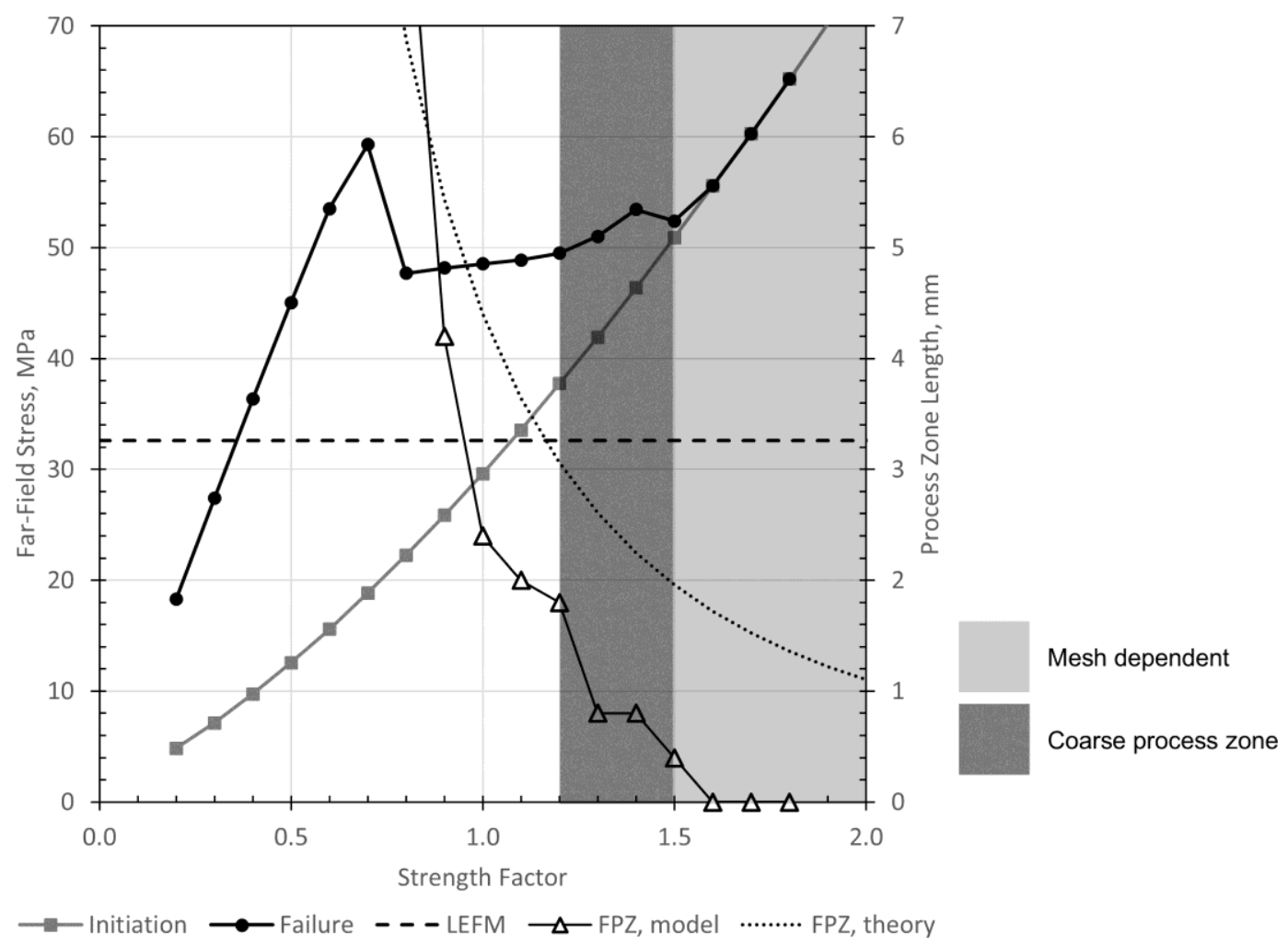

Figure 8. Abaqus CDM model predictions for mode II loading, varying the strength factor.

American Institute of Aeronautics and Astronautics 


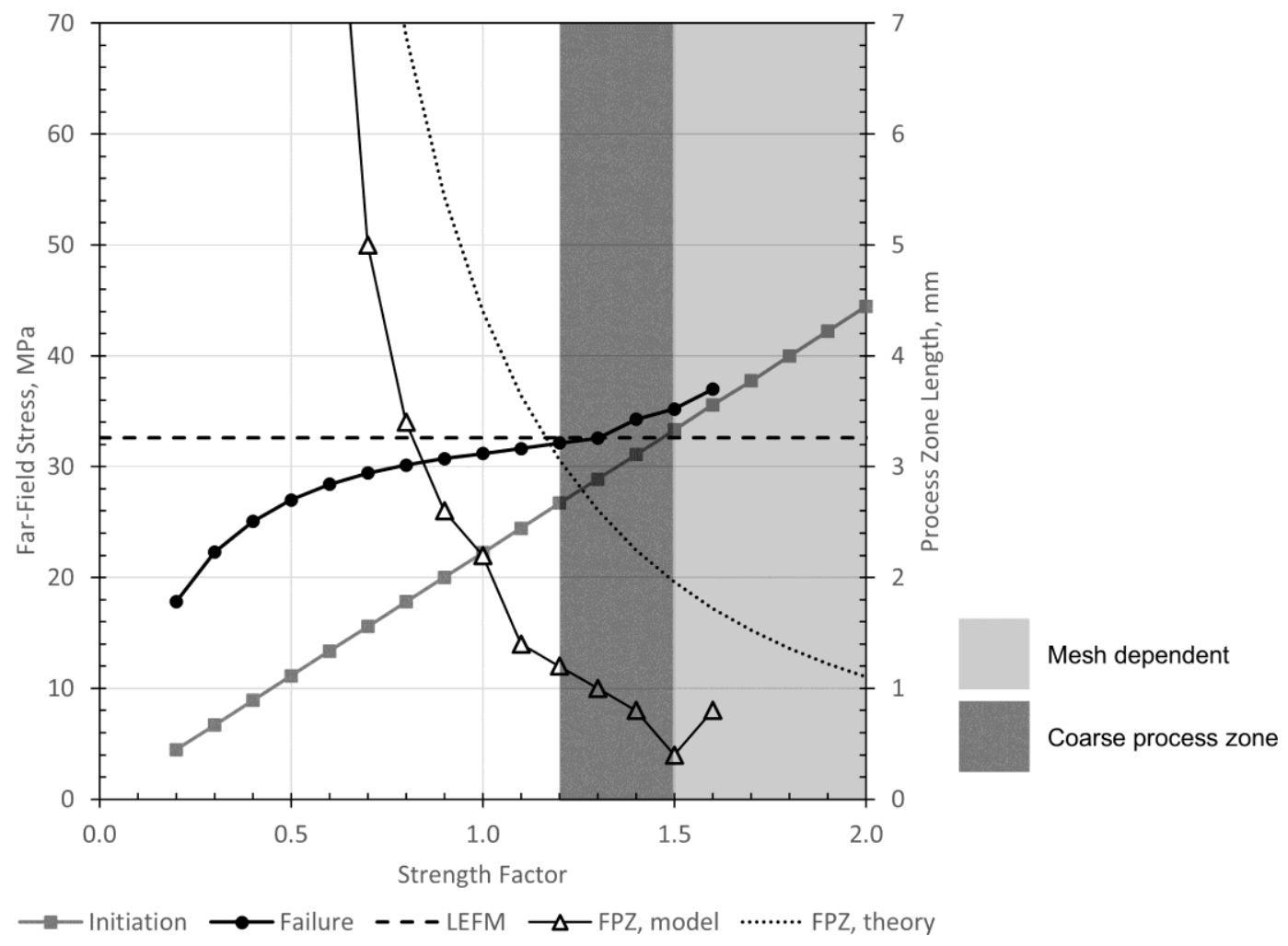

Figure 9. CompDam predictions for mode II loading, varying the strength factor.

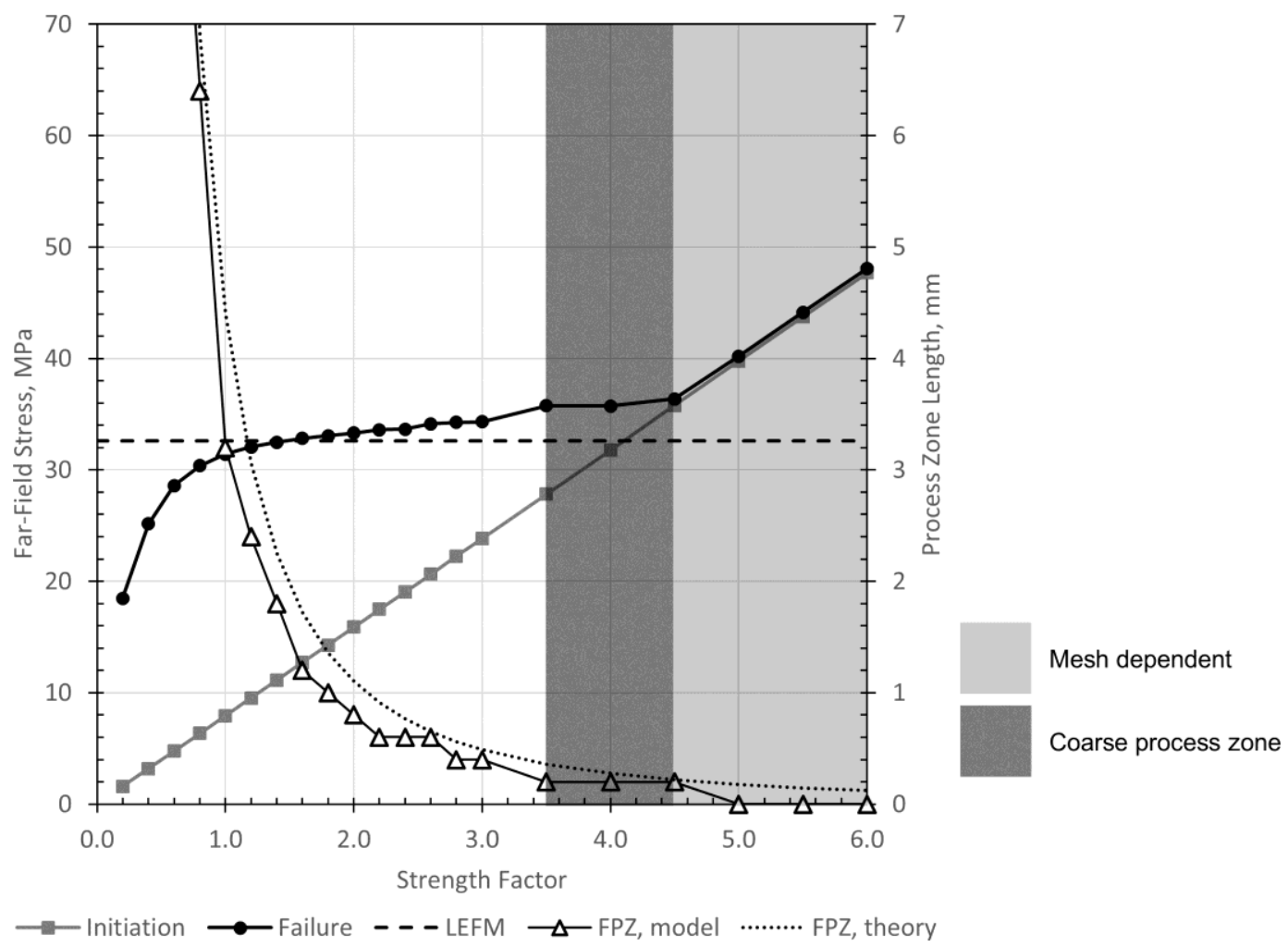

Figure 10. Cohesive element predictions for mode II loading, varying the strength factor. 
Initial crack tip

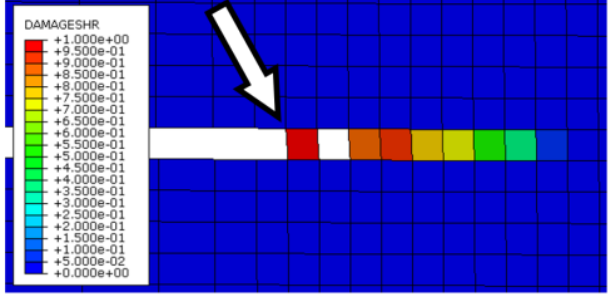

(a)
Initial crack tip

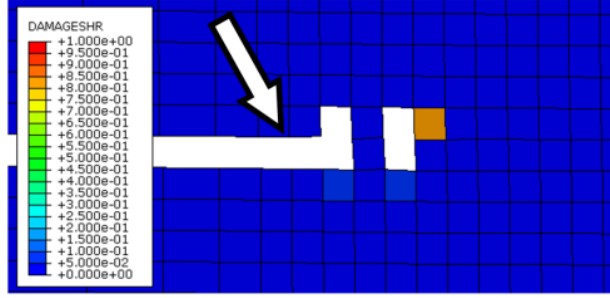

(b)

Figure 11. Close-up view of a crack tip with poor damage propagation behavior: (a) Inner process zone element failure, from the Abaqus CDM strength factor = 1.2 analysis; and (b) Non-self-similar crack growth, from the Abaqus CDM strength factor $=1.9$ analysis. Fully-failed elements are removed for clarity.

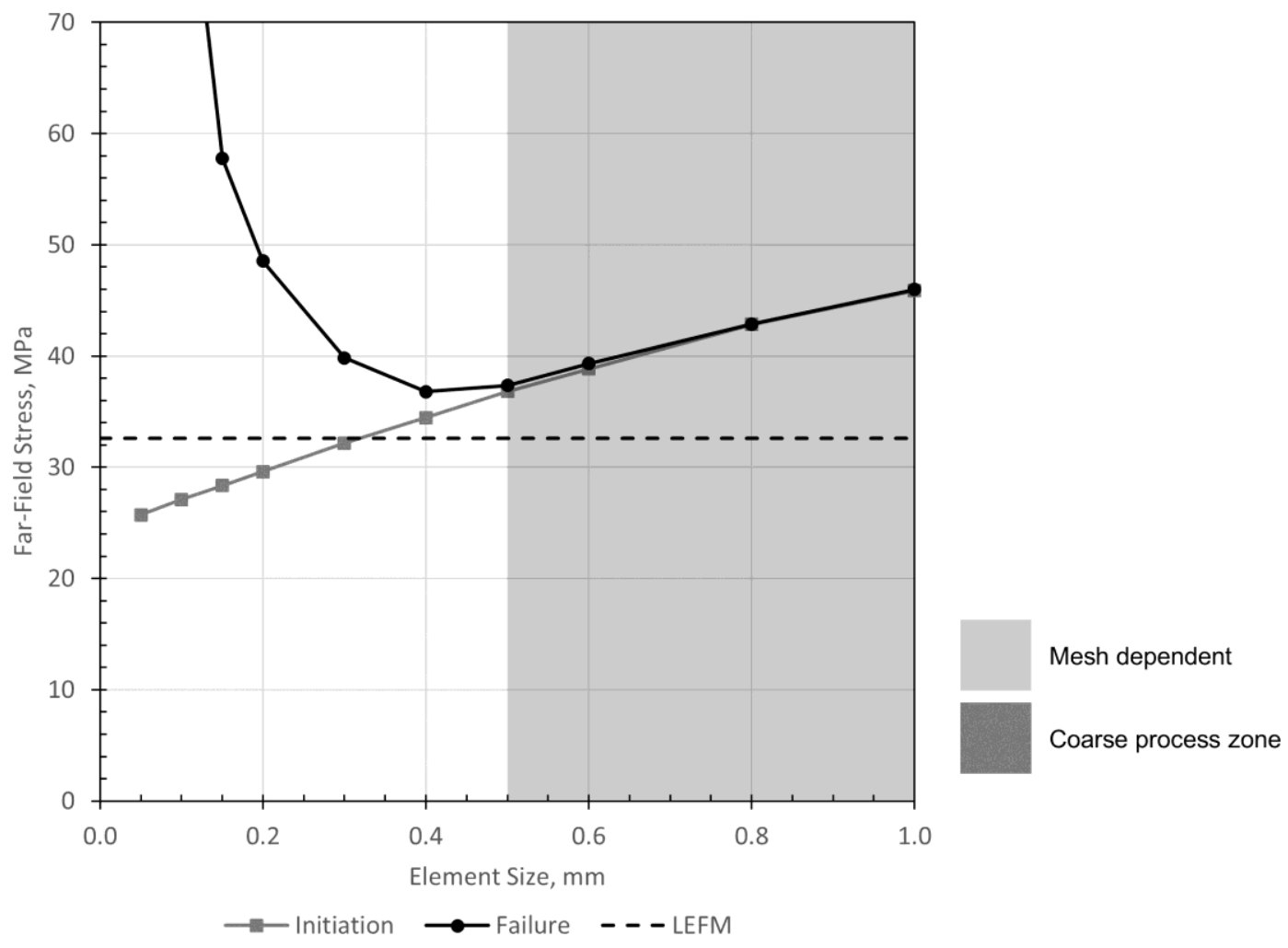

Figure 12. Abaqus CDM predictions for mode II loading, varying the element size. 


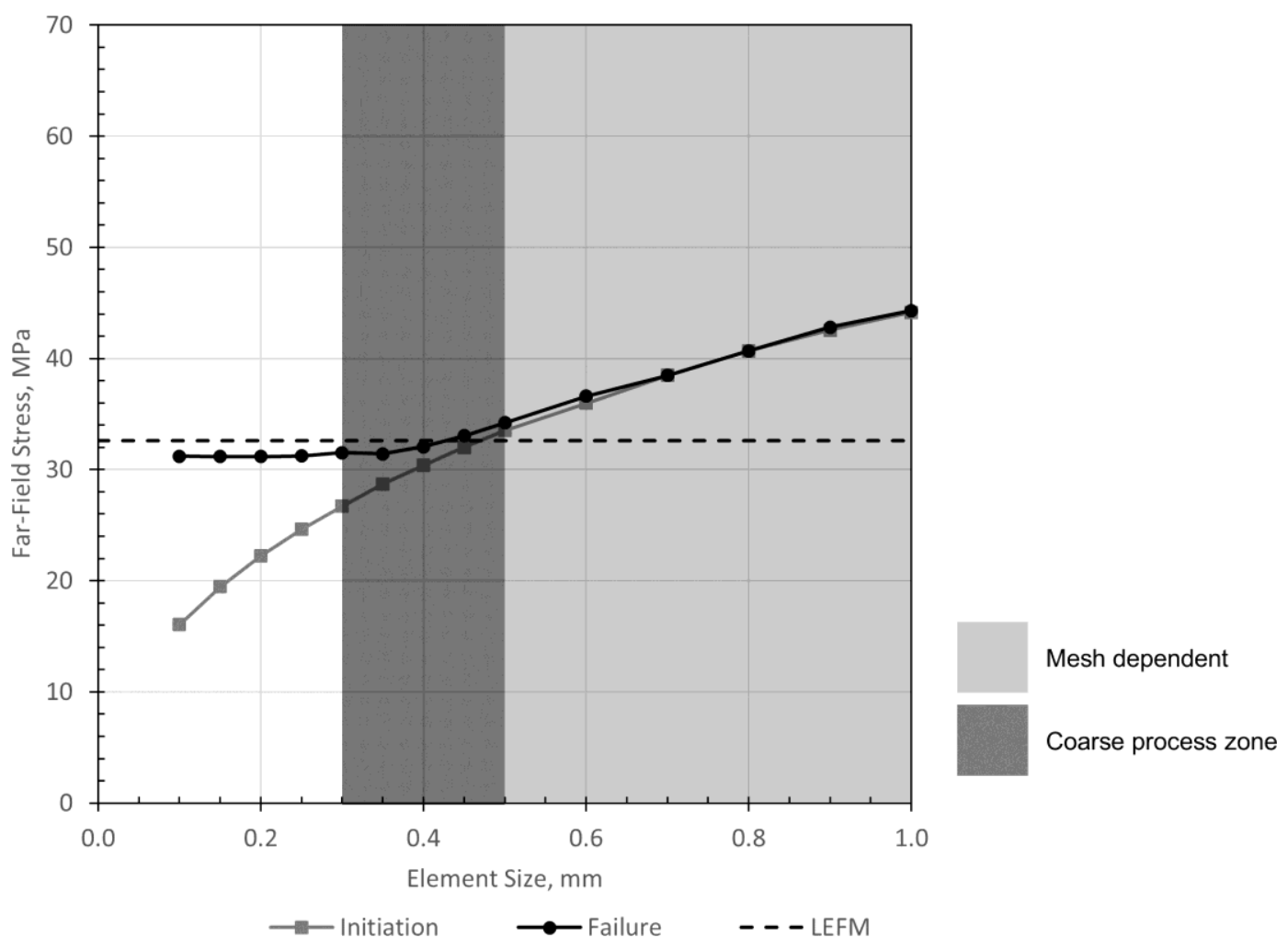

Figure 13. CompDam predictions for mode II loading, varying the element size.

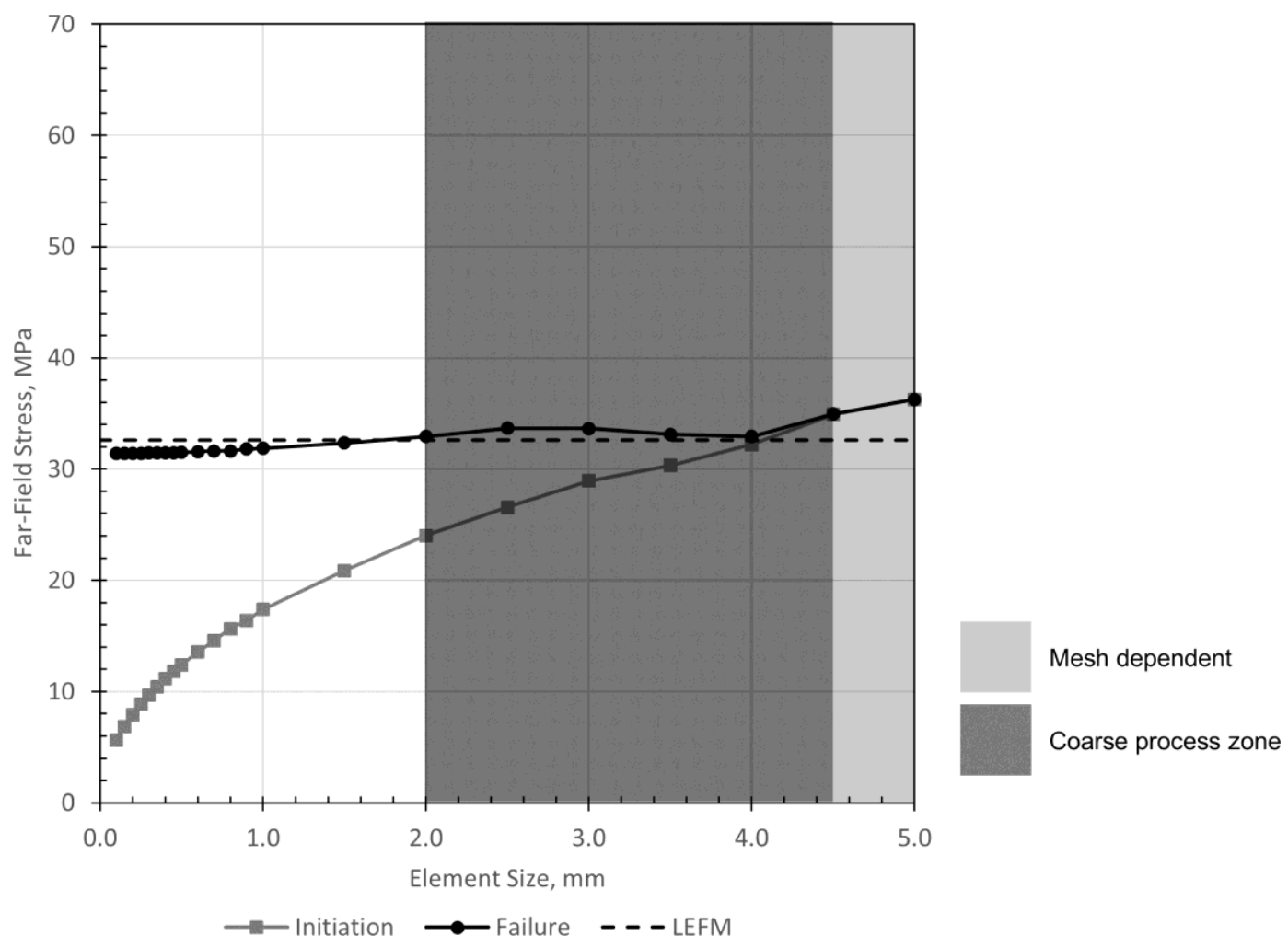

Figure 14. Cohesive element predictions for mode II loading, varying the element size.

American Institute of Aeronautics and Astronautics 